\title{
A novel mutation in JARID1C gene associated with mental retardation
}

\author{
Cristina Santos ${ }^{1,2, \dagger}$, Laia Rodriguez-Revenga ${ }^{1,2, \dagger}$, Irene Madrigal ${ }^{1,2}$, Celia Badenas ${ }^{1,2}$, \\ Merce Pineda ${ }^{3}$ and Montserrat Milà*,1,2 \\ ${ }^{1}$ Genetics Service, Centre de Diagnòstic Biomèdic, Hospital Clínic, Barcelona, Spain; ${ }^{2}$ IDIBAPS (Institut \\ d'Investigacions Biomèdiques August Pi i Sunyer), Barcelona, Spain; ${ }^{3}$ Neurology Service, Hospital Sant Joan de Déu, \\ Barcelona, Spain
}

X-linked mental retardation (XLMR) is an extremely heterogeneous condition that account for $15-25 \%$ of all mentally retarded patients. The number of genes newly reported in relation with this condition has been rapidly increased in the past years. One of the latest is called Jumonji AT-rich interactive domain 1C (JARID1C). This gene encodes for a member of a recently discovered protein family that harbours DNA-binding motifs, suggesting a possible role in transcriptional regulation and in the modification of chromatin structure. In this work we describe the results obtained by screening JARID1C gene in 24 mentally retarded males with history of at least two affected males. Remarkably, we have found a novel missense mutation in exon 10 of the gene that results in a Serine-to-arginine change at amino-acid 451 (S451R). This nucleotide change appears to be restricted to mentally retarded patients, since it has not been detected in control samples. Familial analysis has confirmed the segregation of this mutation with mental retardation. Furthermore, sequence alignment analysis with the different members of the human JARID1 family and with homologous proteins of mouse and fruit fly has revealed that the affected amino acid is conserved. Our data highlights the importance of reporting mutations in this gene since it might support the recent findings that implicates JARID1C with XLMR.

European Journal of Human Genetics (2006) 14, 583-586. doi:10.1038/sj.ejhg.5201608; published online 15 March 2006

Keywords: JARID1C gene; mutational screening; novel mutation; X-linked mental retardation

\section{Introduction}

Mental retardation (MR) is defined as a disability characterized by significant below average intellectual functioning $(\mathrm{IQ}<70)$ in conjunction with significant limitations in adaptative functioning, that occurs before the age of 18 years. ${ }^{1}$ The estimated prevalence of MR is $2-3 \%$ in developed countries. ${ }^{2}$ However, more than $50 \%$ of MR cases remain undiagnosed despite extensive investigation, leaving families without accurate genetic counselling

*Correspondence: Dr M Milà, Genetics Service, Hospital Clínic, C/ Villarroel, 17008036 Barcelona, Spain.

Tel: + 34932275400 ext. 2784; Fax: + 349345152 72;

E-mail: mmila@clinic.ub.es

These two authors contributed equally to this work.

Received 16 November 2005; revised 9 February 2006; accepted 10 February 2006; published online 15 March 2006 or reproductive options, such as prenatal diagnosis. Clinical observations and the study of large families with MR males have highlighted the importance of genes located in the X chromosome. In fact, it is calculated that $\mathrm{X}$-linked mental retardation (XLMR) may account for about $15-25 \%$ of mentally retarded males. ${ }^{3-5}$ To date, the collective efforts of many groups have led to the identification of 59 genes in syndromic and nonsyndromic XLMR. $^{6}$ From all these genes, JARID1C (Jumonji AT-rich interactive domain 1C (MIM 314690)) seems to be one of the more frequently mutated in patients with XLMR, with an estimated frequency of $2-3 \% .^{7}$ This gene, which contains 26 exons, is located in chromosome Xp11.2 region; one of the three $\mathrm{X}$ chromosome intervals in which Ropers et al, ${ }^{8}$ found that approximately $30 \%$ of all mutations underlying nonsyndromic XLMR are clustered. 
The JARID1C gene encodes a member of a conserved protein family, which carries several DNA-binding motifs, suggesting that it is potentially involved in transcriptional regulation and chromatin remodelling. Here, we report a novel JARID1C mutation found in two MR brothers, which cosegregates with the abnormal phenotype in the family.

\section{Materials and methods \\ DNA mutational analysis}

Mutation screening was performed in 24 mentally retarded males that belong to different families with a history of at least two males with significant intellectual impairment. All families were previously examined by a clinical geneticist and or a neuropediatrician who excluded the possibility of a known diagnosis. Moreover, FRAXA and FRAXE syndromes were molecularly ruled out and a normal G-banded karyotype was also obtained in all of them. Genomic DNA was extracted and the complete JARID1C coding sequence was amplified as described by Jensen et al. ${ }^{7}$ Of PCR product, $3 \mu$ l being combined with loading buffer, were denatured and electrophoresed in a $12 \%$ nondenaturing acrylamide for $2 \mathrm{~h}$ at $15-5^{\circ} \mathrm{C}$ using the single-strand conformation polymorphism analysis (SSCP) by genephor (Pharmacia Biotech, Uppsala, Sweden) and following the manufacturer's instructions. When an aberrant migration pattern was detected, PCR amplification product was directly sequenced on an automated sequencer (ABI 3100; Applied Biosystems, Foster City, CA, USA). All the variants detected were further analysed by segregation analysis in the family and/or by screening 250 unrelated control X-chromosomes from Caucasian population.

Blood samples and photographic material from the patients in which a mutation was detected and their family (mother, father, two sisters and one brother) were collected after informed consent.

\section{Online prediction programs}

The SIFT program (http://blocks.fhcrc. org/sift/SIFT.html) uses sequence homology to predict whether an amino-acid substitution will affect protein function and hence, potentially alter phenotype. ${ }^{9}$ Results are reported as 'deleterious or not' according to scores. The PolyPhen program (http://genetics.bwh.harvard.edu/pph) uses the sequence homology and the mapping of the substitution site to known protein three-dimensional structures. ${ }^{10}$ In this case, results are given as 'benign', 'possibly damaging', 'probably damaging', or 'unknown'.

\section{Results}

Mutational analysis of the JARID1C gene in our 24 patients affected of MR allowed us the detection of three different sequence changes. The first one is located in the $5^{\prime}$ UTR region of the gene (c. $-239 \mathrm{G} \rightarrow \mathrm{A}$ ) and was also reported by Jensen et al, ${ }^{7}$ who did not find it in a group of 312 control $\mathrm{X}$ chromosomes. On the basis of these results, the clinical repercussion of this sequence change is uncertain. The second change is a previously described polymorphism located in intron 4 of the JARID1C gene $($ c. $522+19 \mathrm{G} \rightarrow \mathrm{A}){ }^{7}$ Finally, we have also identified a novel missense mutation in exon 10 of the gene (c.1353C > G) in one of the patients also and in his mentally retarded brother. This nonconservative substitution replaces a serine with an arginine (S451R). We have used two online programs, SIFT and PolyPhen to predict a potential effect on protein function for this change. 9,10 The variant was classified as 'deleterious' and proposed to be possibly damaging by both programs, respectively. Moreover, the screening of $250 \mathrm{X}$ chromosomes from unrelated normal Caucasian male population reveals that this change appears to be restricting to patients, since it is absent in controls. Furthermore, familial analysis evidenced the segregation of this mutation with MR and that the mother was also carrying the mutation in heterozygousis. None of the two sisters inherited the putative mutation so theoretically there is no risk for their offspring (Figure 1). Both probands were, respectively, born at term as the fourth and fifth children of healthy unrelated parents with no MR history in their families. They are two boys of 16- and 11-year-old that were referred 10 years ago for fragile X syndrome (FXS) to our molecular laboratory. Apart from the severe MR (IQ 40-50) they also show mild dysmorphisms, including large ears with raised lobe, big hands with large fingers and proximal thumb, prominent and separated superior incisives, scrotal tongue, pectus excavatum, and cubitus valgus (Figure 2). Both have an overfriendly and anxiety character. Metabolic screening, CTscan and EEG performed 6 years ago were normal. Neurological examination did not show any focality or other abnormality and somatic growth was according to their age.

\section{Discussion}

The JARID1C gene, formerly known as 'SMCX', has been recently found to cause XLMR. ${ }^{7}$ It encodes a protein that belongs to the highly conserved ARID (AT-rich interaction domain) family of DNA-binding proteins. Although the biological implication of this protein family is not yet clear, it has been demonstrated that they play roles in transcriptional regulation and are supposed to be involved in the modification of chromatin structure. ${ }^{11,12}$ Similarly to MECP2, XNP, RSK2, ZNF41, and ZNF81, the JARID1C gene belongs to a group of MRX genes that encode proteins with a role in the control of gene expression through modulating the chromatin structure. ${ }^{5}$

To date only one mutational screening of the JARID1C gene has been performed among MR patients suggesting 


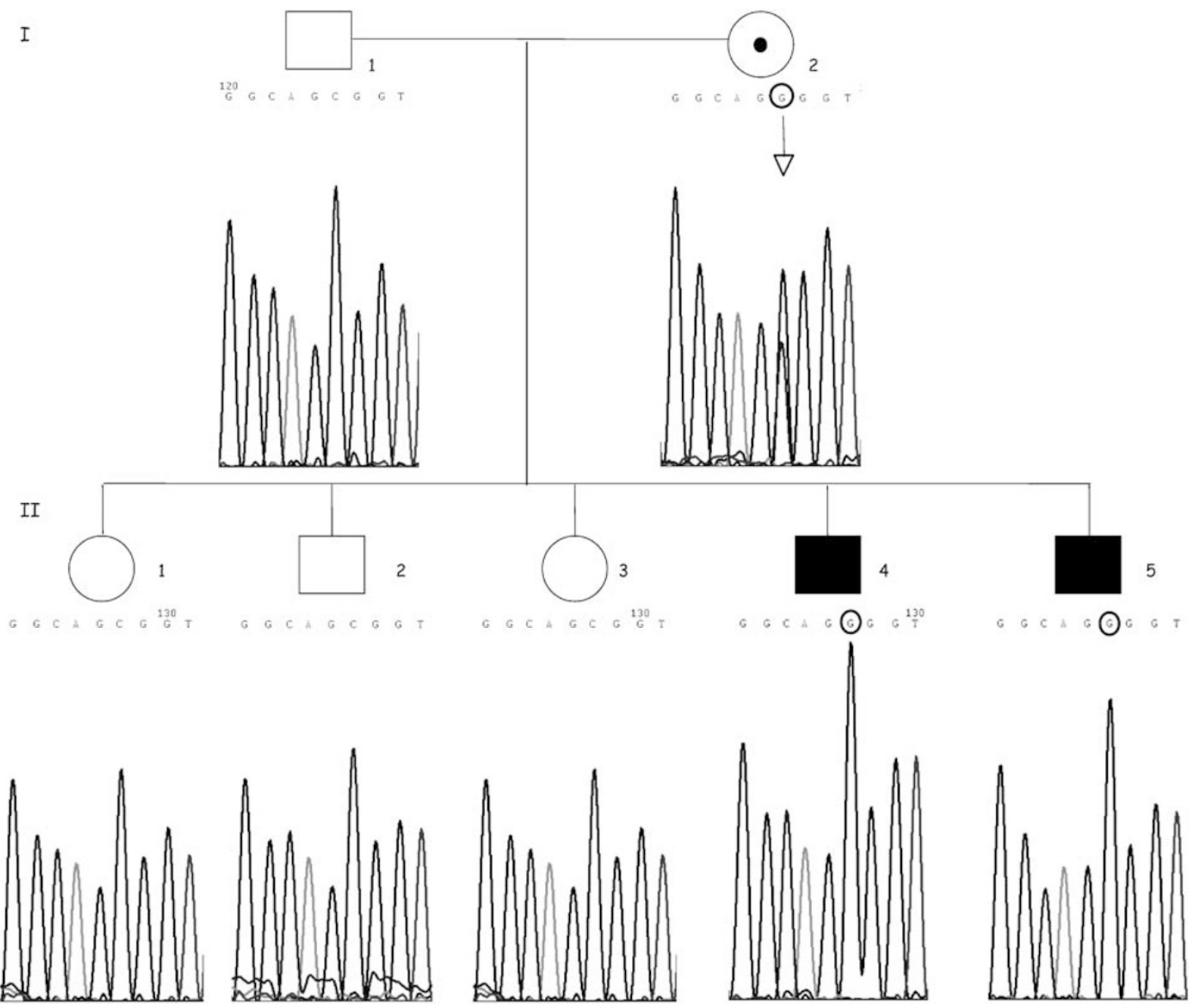

Figure 1 Pedigree of the family and electropherograms of part of exon 10 of the JARID1C gene. The c.1353C > G nucleotide change is indicated with a circle.

that this gene may has an important role in the aetiology of XLMR. ${ }^{7}$ Our data seems to reinforce these results since we have detected a novel missense mutation (S451R) in a family in which it segregates with MR. We hypothesised that $\mathrm{S} 451 \mathrm{R}$ is a disease-causing mutation since it has not been detected among unrelated control samples and it is not present in unaffected members of patients' family. This missense mutation, as the similar ones reported by Jensen et $a l^{7}$ affects an amino acid which is identical in the different members of the human JARID1 family and in homologous proteins of mouse and fruit fly (Figure 3$)^{7}$ Although S451R is not located in one of the conserved JARID1C domains described and we cannot completely exclude the possibility of a rare polymorphism, the replacement of a serine by an arginine alters the charge of the protein and therefore, it may change its threedimensional structure. ${ }^{13}$ Moreover, the employment of online prediction programs used to assess the impact of mutations has classified this S451R variant as possibly damaging. On the basis of all these results, we infer that the S451R mutation interferes with the correct function of the protein and that is the responsible of the phenotype that our patients present. Although clinical data of these patients are scarce there are several features including microcephaly, strabismus, hypermetropia, diastema of teeth, and behavioural or mood problems, that are shared in all of them. The description of these clinical data is of interest since JARID1C mutations have been reported in both syndromic and nonsyndromic XLMR cases. We highlight the importance of reporting them in order to clarify if there is a combination of dysmorphic features that will indicate for a JARID1C mutational screening. Although this kind of mutational analysis are time consuming, and only reveal mutations to a limited number of families they are required since they enhance the diagnostic possibilities and improve the genetic counselling. 



Figure 2 Phenotypic aspect of the two affected brothers of the family with the S451R mutation. Note the large fingers with very proximal thumbs and the scrotal tongue.

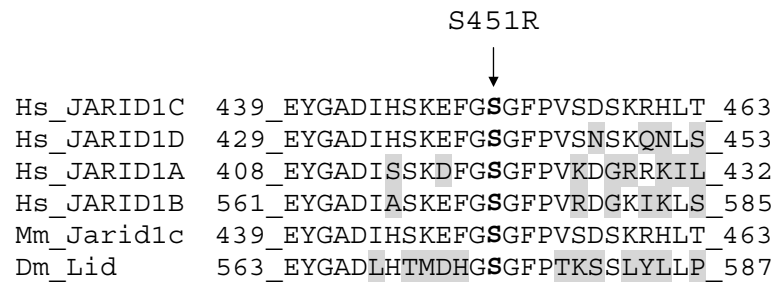

Figure 3 Partial amino-acid sequence alignment of the members of the human JARID1 family (Hs_JARID1A-Hs_JARID1D), the JARID1C orthologous protein of mouse (Mm_Jarid1c), and the homologous Lid protein of Drosophila melanogaster (Dm_Lid). The missense mutation detected and its position is indicated by an arrow and in bold letter. Amino acids different from the JARID1C sequence are in grey.

\section{Acknowledgements}

We thank the family for their cooperation and consent to publish this study and their photographs. This work has received financial support from GIRMOGEN (V2003REDG 03-98), REDGEN (V2003REDC 07), INERGEN (C03/05), PI050776, PIO50159, and V2004-FSO41126-O financed by the 'Fondo de Investigación Sanitaria', Spain.

\section{References}

1 American Association on Mental Retardation. Mental Retardation: Definition, Classification, and Systems of Supports, 10th edn. Washington 2002.
2 Leonard $\mathrm{H}$, Wen $\mathrm{X}$ : The epidemiology of mental retardation: challenges and opportunities in the new millennium. Ment Retard Dev Disabil Res Rev 2002; 8: 117-134.

3 Fishburn J, Turner G, Daniel A, Brookwell R: The diagnosis and frequency of X-linked conditions in a cohort of moderately retarded males with affected brothers. Am J Med Genet 1983; 14: $713-724$.

4 Turner G: Finding genes on the $\mathrm{X}$ chromosome by which homo may have become sapiens. Am J Hum Genet 1996; 58: 1109-1110.

5 Kleefstra T, Hamel BC: X-linked mental retardation: further lumping, splitting and emerging phenotypes. Clin Genet 2005; 67: $451-467$.

6 Ropers HH, Hamel BC: X-linked mental retardation. Nat Rev Genet 2005; 6: 46-57.

7 Jensen LR, Amende M, Gurok U et al: Mutations in the JARID1C gene, which is involved in transcriptional regulation and chromatin remodeling, cause X-linked mental retardation. Am J Hum Genet 2005; 76: 227-236.

8 Ropers HH, Hoeltzenbein M, Kalscheuer V et al: Nonsyndromic Xlinked mental retardation: where are the missing mutations? Trends Genet 2003; 19: 316-320.

$9 \mathrm{Ng}$ PC, Henikoff S: SIFT: predicting amino acid changes that affect protein function. Nucleic Acids Res 2003; 31: 3812-3814.

10 Ramensky V, Bork P, Sunyaev S: Human non-synonymous SNPs: server and survey. Nucleic Acids Res 2002; 30: 3894-3900.

11 Kortschak RD, Tucker PW, Saint R: ARID proteins come in from the desert. Trends Biochem Sci 2000; 25: 294-299.

12 Patsialou A, Wilsker D, Moran E: DNA-binding properties of ARID family proteins. Nucleic Acids Res 2005; 33: 66-80.

13 Marchler-Bauer A, Anderson JB, DeWeese-Scott C et al: CDD: a curated Entrez database of conserved domain alignments. Nucleic Acids Res 2003; 31: 383-387. 